\title{
A HE-GAS COOLED, STATIONARY GRANULAR TARGET
}

\author{
P. Pugnat and P. Sievers
}

In the CERN approach to the design of a neutrino factory, the repetition frequency of the proton beam is high enough to consider stationary solid targets as a viable solution for multi-MW beams. The target consists of high density tantalum spheres of $2 \mathrm{~mm}$ diameter which can efficiently be cooled by passing a high mass flow He-gas stream through the voids between the Ta-granules. Very small thermal shocks and stresses will arise in this fine grained structure due to the relatively long burst of $3.3 \mu$ s from the SPL-proton linac. In a quadruple target system where each target receives only one quarter of the total beam power of $4 \mathrm{MW}$, conservative temperature levels and adequate lifetimes of the target are estimated in its very high radiation environment. A conceptual design of the integration of the target into the magnetic horn-pion-collector is presented.

LHC Division

$$
\begin{gathered}
\text { Presented at the 4th NuFact'02 Workshop } \\
\text { 1-6 July 2002, London, UK } \\
\text { and } \\
\text { To be published in the "Journal of Physics G" }
\end{gathered}
$$

Administrative Secretariat LHC Division CERN

CH - 1211 Geneva 23
Geneva, Switzerland

6 January 2003 


\title{
A He-gas cooled, stationary granular target
}

\author{
P. Pugnat and P. Sievers \\ CERN, Geneva, Switzerland
}

\begin{abstract}
In the CERN approach to the design of a neutrino factory, the repetition frequency of the proton beam is high enough to consider stationary solid targets as a viable solution for multi-MW beams. The target consists of high density tantalum spheres of $2 \mathrm{~mm}$ diameter which can efficiently be cooled by passing a high mass flow He-gas stream through the voids between the Ta-granules. Very small thermal shocks and stresses will arise in this fine grained structure due to the relatively long burst of $3.3 \mu$ s from the SPL-proton linac. In a quadruple target system where each target receives only one quarter of the total beam power of $4 \mathrm{MW}$, conservative temperature levels and adequate lifetimes of the target are estimated in its very high radiation environment. A conceptual design of the integration of the target into the magnetic horn-pion-collector is presented.
\end{abstract}

PACS numbers: 29.25.-t

\section{Introduction}

Free liquid mercury jets are considered for the target of future Neutrino Factories [1]. However, in view of the considerable technical challenges and developments required for such a solution [2], alternatives like moving [3] or stationary solid targets [4] are considered in parallel. The latter is made out of small, high density tantalum or tungsten carbide spheres and is cooled either by water or gaseous helium. Similar approaches have been made for the targets of neutron spallation sources [5]. This type of target seems particularly suited for the targeting of the CERN-SPL proton source [6] where beam bursts of $2.2 \mathrm{GeV} / \mathrm{c}$ protons with a duration of $3.3 \mu \mathrm{s}$ each are impinging on the target at a repetition rate of $50 \mathrm{~Hz}$, much higher than for synchrotron driven beams. At an average beam power of $4 \mathrm{MW}$ this leads therefore to smaller adiabatic temperature rises of about $100 \mathrm{~K}$ per burst. The target is operated in a regime close to an equivalent continuous heat input source where the resulting average temperatures are dominated mainly by the efficiency of the cooling system. This is favourably supported by the high surface to volume ratio of the ensemble of granules with a diameter of around $2 \mathrm{~mm}$, which are exposed to the cooling medium flowing between them. Moreover, the adiabatic temperature increase per burst rising during $3.3 \mu \mathrm{s}$ is slow compared to the characteristic time constant of $0.26 \mu$ s over which vibrations and dynamic stresses can be induced inside these small spheres [7].

\section{He-Gas Cooling}

Each proton burst deposits a total energy of $16 \mathrm{~kJ}$ in the target with a maximum energy density of $15 \mathrm{~J} / \mathrm{g}$ along its axis [8]. This leads to adiabatic temperature spikes along this location with an amplitude of $\Delta T=100 \mathrm{~K}$ at a frequency of $50 \mathrm{~Hz}$. Between the spikes, the temperature of the spheres decays exponentially over $20 \mathrm{~ms}$ with a time constant which is essentially given by the efficiency of the He-cooling, from a peak $T_{S, \text { Max }}$ to a valley temperature $T_{S, \min }$ before rising again adiabatically by $100 \mathrm{~K}$ due to the following proton 
burst. This has been assessed in more detail in [9] assuming a He-gas stream at a pressure of $20 \mathrm{~atm}$ and a velocity of $100 \mathrm{~m} / \mathrm{s}$ which results in a total mass flow $\dot{m}_{\mathrm{He}}=0.36 \mathrm{~kg} / \mathrm{s}$. From this, together with the target geometry, a convection coefficient for the cooling of $10 \mathrm{~kW} / \mathrm{m}^{2} \mathrm{~K}$ is derived, leading finally to a time constant of $136 \mathrm{~ms}$. The results are summarized in Table 1 for the $4 \mathrm{MW}$ beam where in addition to the target temperatures also the peak and valley temperatures $T_{H e, M a x}$ and $T_{H e, \min }$ of the helium at its exit are given for two mass flows. These rather elevated temperatures can be reduced to very conservative values by using a quadruple target system as proposed in [10]. There one out of four targets is hit in turn by every fourth proton burst thus reducing the average power on each target by a factor of four.

Table 1: Minimum and maximum temperature inside spheres and He-gas during the $50 \mathrm{~Hz}$ pulsed beam. The time average temperatures refer to a $4 \mathrm{MW}$ continuous beam.

\begin{tabular}{lcccccc}
\hline & $\mathrm{T}_{\mathrm{S}, \min }$ & $\mathrm{T}_{\mathrm{S} . \text { Max }}$ & $\mathrm{T}_{\mathrm{S} \text {.average }}$ & $\mathrm{T}_{\mathrm{He}, \min }$ & $\mathrm{T}_{\mathrm{He}, \text { Max }}$ & $\mathrm{T}_{\text {He.average }}$ \\
\hline Single target, $\dot{m}_{\mathrm{He}}=0.36 \mathrm{~kg} / \mathrm{s}$ & 631 & 731 & 680 & 505 & 585 & 544 \\
Single target, $\dot{m}_{\mathrm{He}}=0.72 \mathrm{~kg} / \mathrm{s}$ & 387 & 487 & 435 & 240 & 302 & 270 \\
Quadruple target, $\dot{m}_{\mathrm{He}}=0.36 \mathrm{~kg} / \mathrm{s}$ & 125 & 225 & 170 & 100 & 180 & 136 \\
\hline
\end{tabular}

\section{Technical Solutions and Integration}

In addition to the mechanical integrity and to the cooling of a stationary target, its containment and its radiation damage has to be considered. The most critical item in this respect is the beam entrance window separating the beam vacuum from the target and in particular from its cooling medium. There, the energy density is comparable to that in the target, i.e. a temperature rise of about $40 \mathrm{~K} /$ pulse will be reached in a Titanium window. Its time average peak power at the beam center will again be of the order of $1 \mathrm{~kW} / \mathrm{g}$. Considering a window thickness of $0.1 \mathrm{~mm}$, this amounts to a surface power of $50 \mathrm{~W} / \mathrm{cm}^{2}$ to be evacuated through only one side of the window. With the above assumed He-cooling circuit, this will lead to a time average temperature rise of about $50 \mathrm{~K}$. Adding to this the adiabatic temperature rise due to each proton burst the beam window will operate well above $100^{\circ} \mathrm{C}$ at its hottest spot. Windows made out of beryllium will reach lower temperatures, but are not considered here. Again, as for the quadruple target, distributing the beam power over four target-window units would clearly be beneficial and would increase their life time accordingly.

Considering radiation damage of the beam entrance window, data are available for proton beams of $800 \mathrm{MeV}$ [11]. Scaling from these energies and proton fluencies to the CERNSPL-parameters, as a first approximation about 10 dpa's (displacements per atom) per week are estimated. This clearly underlines again that a rapid exchange of the beam window or, even better, applying the quadruple target scheme must be envisaged.

Bearing the above developed arguments in mind, it is proposed to integrate the granular stationary target into the magnetic horn collector as shown in Fig.1. The granules are contained in a radiation hard, metallic wire net confining the spheres, but being transparent to the cooling gas. This is guided via anular inlet and outlet ducts laterally across the target cylinder as shown in Fig.1. These pipes have still to be optimised in terms of cross section and wall thickness to reduce the gas velocity in this part while increasing the transparency for pions traversing this region. The entrance and exit of the target are also covered by a 
transparent wire net which has no structural function and must only retain the Ta-spheres in place. The actual beam entrance window is moved further upstream, away from the "hot" area of the target where the beam spot is larger and which causes less local damage and heating. As shown in Fig.1, this additional volume will be filled by nearly static, but high pressure (20 atm) Helium. An auxiliary circuit is envisaged to blow gas directly against the inner surface of the window to cool the beam heated area.

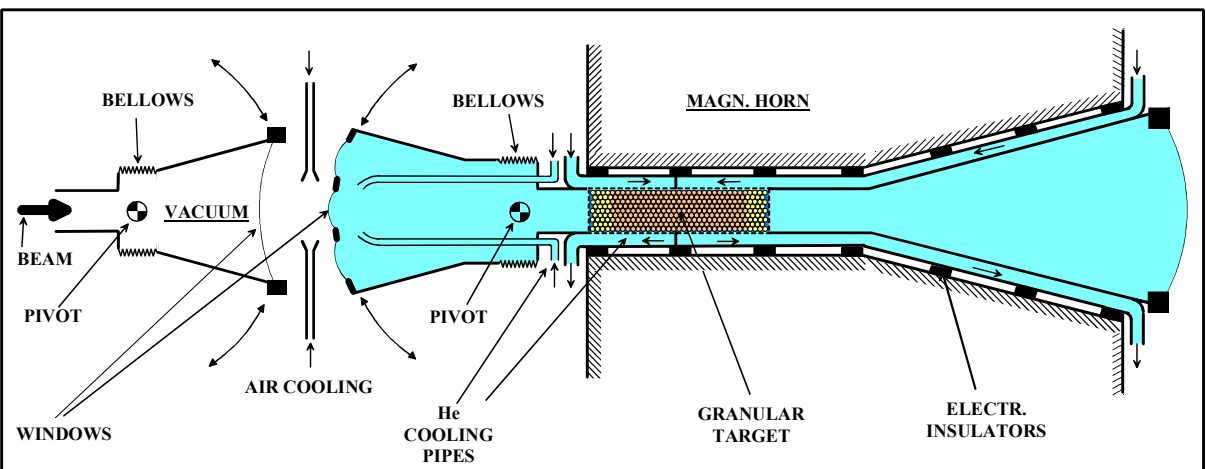

Figure 1: The granular, stationary target, its He-gas cooling system and its pivoting windows integrated into the magnetic horn-pion-collector.

To prolongate the lifetime of the window, one may pivot azimuthally via a bellows system the upstream pipe which is enlarged at its entrance to allow for the installation of $3-6$ windows of which each can be moved into the beam line by remote control. This should extend the safe running time of the unit to several months. In principle, the upstream target window could face directly the beam vacuum. In Fig.1, a solution is sketched where the beam vacuum is closed by its proper window and again made mobile to prolongate its lifetime. In this case, the 20 atm helium volume is mechanically separated from the beam vacuum. This allows for additional air cooling between both windows. At the downstream side a similar extension of the high pressure gas volume is proposed where however the local energy density on the downstream window is much reduced and which should not require any remote displacement mechanism to distribute the radiation dose.

\section{References}

[1] Palmer R B et al 2000 NIM-A 451 265-278.

[2] Lettry J Review of target developments for a neutrino factory, these proceedings.

[3] Bennet J R J et al 2000 NIM-A 451 344-348, Thieberger P A and Kirk H G 2002 BNL-MUC-Note-Target-234.

[4] Sievers P 2001 A stationary target for the CERN-neutrino factory, CERN-NuFact-Note-065 and Proceedings of NuFact-01 (Tsukuba), NIM-A (to be published).

[5] Conrad H 1994 ESS-Juelich-Internal-Note, Ammerman C et al 2001 ANS Winter Annual Meeting, (Reno, Nevada).

[6] Garoby R $200220^{\text {th }}$ ICFA Advanced Beam Dynamics Workshop, FNL, Illinois.

[7] Sievers P and Pugnat P 2000 CERN-NuFact-Note-035.

[8] Gilardoni S 2001 CERN-PS, private communication.

[9] Pugnat $P$ and Sievers P, to be published.

[10] Autin B and Verdier A 2001 CERN-NuFact-Note-100 and Proceedings of NuFact-01 (Tsukuba), NIM-A (to be published).

[11] Gromov B E et al, and Dedoul A et al 2001 Proceedings of ANS Winter Annual Meeting, (Reno, Nevada). 